\title{
Interactions of Streptococcus suis serotype 2 with human meningeal cells and astrocytes
}

\author{
Jean-Philippe Auger ${ }^{1}$, Myron Christodoulides², Mariela Segura ${ }^{1}$, Jianguo Xu ${ }^{3}$ and Marcelo Gottschalk*
}

\begin{abstract}
Background: Streptococcus suis serotype 2 is an important porcine pathogen and emerging zoonotic agent responsible for meningitis, of which different sequence types predominate worldwide. Though bacterial meningitis is defined as an exacerbated inflammation of the meninges, the underlying astrocytes of the glia limitans superficialis may also be implicated. However, the interactions between this pathogen and human meningeal cells or astrocytes remain unknown. Furthermore, the roles of well-described virulence factors (capsular polysaccharide, suilysin and cell wall modifications) in these interactions have yet to be studied. Consequently, the interactions between S. suis serotype 2 and human meningeal cells or astrocytes were evaluated for the first time in order to better understand their involvement during meningitis in humans.

Results: Streptococcus suis serotype 2 adhered to human meningeal cells and astrocytes; invasion of meningeal cells was rare however, whereas invasion of astrocytes was generally more frequent. Regardless of the interaction or cell type, differences were not observed between sequence types. Though the capsular polysaccharide modulated the adhesion to and invasion of meningeal cells and astrocytes, the suilysin and cell wall modifications only influenced astrocyte invasion. Surprising, S. suis serotype 2 induced little or no inflammatory response from both cell types, but this absence of inflammatory response was probably not due to $S$. suis-induced cell death.

Conclusions: Though S. suis serotype 2 interacted with human meningeal cells and astrocytes, there was no correlation between sequence type and interaction. Consequently, the adhesion to and invasion of human meningeal cells and astrocytes are strain-specific characteristics. As such, the meningeal cells of the leptomeninges and the astrocytes of the glia limitans superficialis may not be directly implicated in the inflammatory response observed during meningitis in humans.
\end{abstract}

Keywords: Streptococcus suis serotype 2, Pig pathogen, Zoonosis, Meningeal cells, Astrocytes, Meningitis

\section{Background}

Streptococcus suis is an important porcine bacterial pathogen and emerging zoonotic agent responsible for sepsis and meningitis [1]. Of the thirty-five described serotypes, based on the presence of the capsular polysaccharide (CPS) or its respective genes, serotype 2 is

\footnotetext{
*Correspondence: marcelo.gottschalk@umontreal.ca

${ }^{1}$ Faculty of Veterinary Medicine, Research Group on Infectious

Diseases of Swine (GREMIP) and Swine and Poultry Infectious Diseases

Research Center (CRIPA), University of Montreal, 3200 Sicotte Street,

Saint-Hyacinthe, QC J2S 2M2, Canada

Full list of author information is available at the end of the article
}

regarded as the most widespread and virulent, responsible for the majority of porcine and human cases of infection worldwide [2]. In fact, $97 \%$ of the approximately 1300 serotyped S. suis cases of infection in humans were caused by the serotype 2 , of which nearly $70 \%$ presented clinical signs of meningitis [2]. Furthermore, this pathogen is the first cause of adult bacterial meningitis in Vietnam, the second most common in Thailand, and the third most frequent cause of community-acquired bacterial meningitis in Hong Kong [2]. The use of multilocus sequence typing has identified four predominate serotype 2 sequence types (ST): the ST1 in Eurasia, the ST7, 
responsible for the human outbreaks of 1998 and 2005, in China, and the ST25 and ST28 in North America [2]. In addition, porcine and human cases of infection have been attributed to ST25 and ST28 strains in Thailand and Japan, though less frequently [2]. Moreover, differential interactions have been observed between STs using various cell types from different species [3, 4].

Of the multitude of $S$. suis serotype 2 virulence factors described [1], the CPS, suilysin (SLY) and cell wall modifications have been well-characterized. The CPS is a critical factor implicated in a multitude of functions, most importantly in the resistance to phagocytosis $[5,6]$. Meanwhile, the SLY is a hemolysin responsible for causing cell cytotoxicity [7]. Finally, cell wall modifications, such as the D-alanylation of the lipoteichoic acid (LTA) and N-deacetylation of the peptidoglycan (PGN), are known to interfere with the immune response; the D-alanylation of the LTA was also shown to modulate adhesion to and invasion of endothelial cells $[6,8,9]$.

The interactions between $S$. suis serotype 2 and certain cells of the central nervous system (CNS) have been studied, including porcine brain microvascular endothelial cells (pBMEC) and porcine choroid plexus epithelial cells (CPEC), as well as murine microglia and astrocytes $[4,10-12]$. However, few studies have used human cells: only the interactions with human BMEC (hBMEC) and choroid plexus papilloma cells have been reported [13, 14], while those with meningeal cells and other cells of the human CNS remain unknown. The meninges are composed of the dura mater and leptomeninges: the latter are formed of the pia mater and arachnoid mater together with the trabeculae that traverse the cerebrospinal fluid (CSF)-filled subarachnoid space (SAS) [15]. The pia mater overlies the glia limitans superficialis, a layer of compact astrocytes that surrounds the brain and forms a barrier [16]. While astrocytes play a crucial role in cerebral homeostasis, they also participate in inflammation, though this role has only begun to be studied [17, 18]. Consequently, astrocytes possess a multitude of pattern recognition receptors involved in the innate immune response, including Toll-like receptors (TLRs) and nucleotide-binding oligomerization domain (NOD)-like receptors (NLRs) [18]. As such, these cells are a source of certain inflammatory mediators [18].

Penetration of the CPEC that form the blood-CSF barrier by $S$. suis, from the underlying blood vessels, is a proposed portal of entry into the CNS $[14,19]$. Consequently, the leptomeninges and underlying astrocytes of the glia limitans are possibly implicated in both porcine and human S. suis infections, which are characterized by meningitis (inflammation of the leptomeninges) often accompanied by inflammation of the surrounding CNS tissues, such as the glia limitans [20]. The interactions of different important meningitis-causing human bacterial pathogens with these cells have already been studied [21-24].

In this study, the interactions between different $S$. suis serotype 2 strains and human meningeal cells or astrocytes, including certain well-characterized virulence factors, were evaluated for the first time in order to better understand the implication of the meninges and underlying CNS tissue during meningitis in humans.

\section{Results and discussion}

Streptococcus suis serotype 2 adheres to but rarely invades human meningeal cells and these interactions are modulated by the CPS

Though a high blood bacterial load is often regarded as crucial for the development of meningitis by extracellular bacterial pathogens (eg. Escherichia coli) [25], this remains unknown for S. suis. Nonetheless, bacteria must breach one of the physiological barriers that separate the brain: the blood-CSF barrier is the preferred site of entry into the CNS for most meningitis-causing human bacterial pathogens, which subsequently reach the CSF and the meningeal cells with which they may interact [19]. Bacterial adhesion to the cell surface is considered a critical host-pathogen interaction. Consequently, the adhesion of the $S$. suis strains, belonging to different STs, to human meningeal cells was measured. Since primary human leptomeningeal cells cannot be reliably cultured, previously established human leptomeningioma cell cultures were used [26]. These cells present the same cytological and morphological structures as their primary counterparts [24] and have been used with various meningitis-causing human bacterial pathogens [21, 23]. The ST1, ST7, ST25, and ST28 strains all adhered to meningeal cells, with associated bacteria ranging between $5 \times 10^{4}$ colony forming units (CFU) and $1 \times 10^{7} \mathrm{CFU}$, equivalent to 1 and 2500 bacteria/cell, respectively (Fig. 1A). Though significant differences were not observed between STs, there were differences between strains: P1/7 and 89-1591 adhered significantly less and more than the other strains $(\mathrm{p}<0.01)$, respectively. These differences suggest the partial implication of strain-specific factors [1], which remain largely unknown since nearly all virulence studies have been conducted using ST1, and more recently, ST7 strains [1]. Consequently, very little is known regarding ST25 and ST28-specific virulence factors, including those implicated in adhesion. The adhesion levels of $S$. suis are similar to those reported for group B Streptococcus (GBS) serotypes III and V, yet S. suis adhered more strongly than other important encapsulated human bacterial pathogens, such as Streptococcus pneumoniae serotype 2, Haemophilus influenzae type 

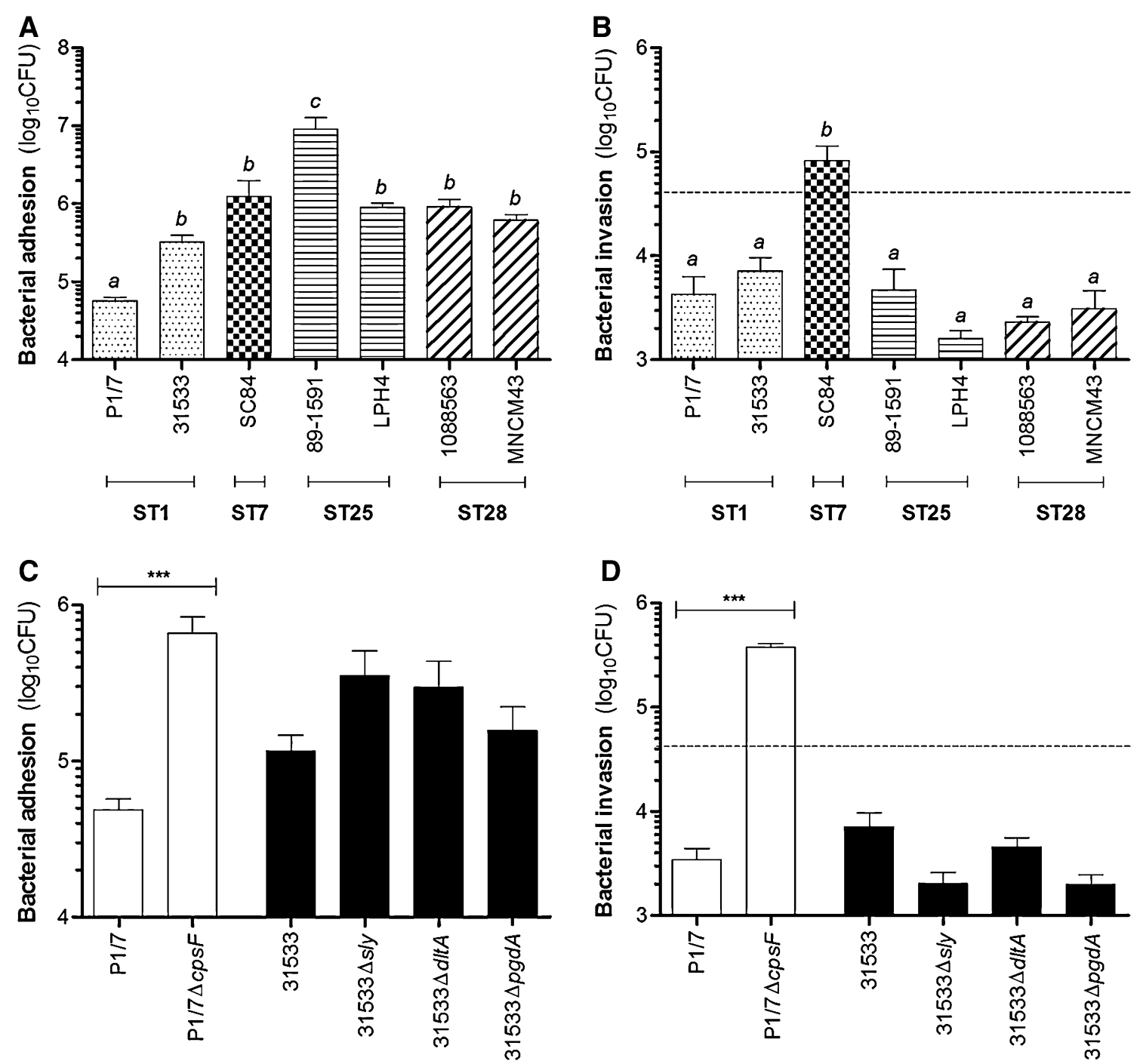

Fig. 1 Interactions of S. suis serotype 2 with human meningeal cells. Bacterial adhesion (A) and invasion (B) of human meningeal cells by the S. suis strains belonging to different sequence types (STs), and adhesion (C) and invasion (D) of human meningeal cells by the mutant strains. Results are expressed as $\log _{10}$ mean \pm SEM CFU obtained from three independent experiments. Dotted lines indicate a ratio of 1 intracellular bacterium/cell. The use of different letters $(a, b$ or $c)$ indicates a significant difference between groups $(p<0.01)$; *** $(p<0.001)$

b and E. coli K1, but less so than Neisseria meningitidis serogroup $B$, when similar initial concentrations were used [21, 23]. This adhesion capacity of S. suis serotype 2 had also been observed when porcine tracheal epithelial cells, human lung epithelial cells, hBMEC or pBMEC were infected with ST1 strains [11, 13, 27, 28]. Importantly, this is the first time that the interactions between S. suis serotype 2 ST25 or ST28 strains and CNS cells, other than the BMECs, have been studied, regardless of the species. Unlike with human and porcine intestinal epithelial cells [29], no differences were observed between adhesion of human or porcine S. suis serotype 2 strains to human meningeal cells.
Since S. suis adhered to meningeal cells, its capacity to subsequently invade the cells was evaluated. Bacterial invasion ranged between $2 \times 10^{3}$ and $7 \times 10^{3} \mathrm{CFU}$ for the different strains, equivalent to 0.05 and 0.2 intracellular bacterium/cell, respectively, with the exception of the ST7 strain, for which $8 \times 10^{4} \mathrm{CFU}$ were recovered, equivalent to 2 intracellular bacteria/cell (Fig. 1B). Unfortunately, the definition of bacterial invasion remains controversial and subjective, with no criterion allowing to determine with certainty if a bacterial strain is invasive [30]. Though the number of internalized bacteria required for $S$. suis serotype 2 to induce meningitis remains unknown, it was previously suggested that 
the survival of a single intracellular $H$. influenzae may result in meningitis [31]. Consequently, biologically relevant invasion was defined as 1 intracellular bacterium/ cell in this study. As such, only the ST7 was capable, in theory, of meningeal cell invasion: this capacity could be the result of unique factors, since important differences have been observed in the genome of this strain when compared to ST1 and ST25 strains [32]. The general inability of $S$. suis to invade meningeal cells is a characteristic shared with GBS, S. pneumoniae and $H$. influenzae $[21,23]$. By contrast, $N$. meningitidis and $E$. coli invaded meningeal cells [23]. It was previously demonstrated that though S. suis serotype 2 invaded porcine tracheal epithelial cells, it did not invade human lung epithelial cells or hBMEC; invasion of pBMEC remains controversial however $[11,13,27,28,30]$. Breaching of the meningeal cell barrier via the paracellular route cannot be discarded however, since this route is difficult to evaluate in the absence of transwell inserts and measuring of the transepithelial electrical resistance. Although described for intestinal epithelial cells [29], this route of entry has not yet been described for $S$. suis in the CNS, where bacteria were reported to use the transcellular route (cellular invasion) $[11,14,33]$. Surprisingly, a correlation between meningeal cell adhesion and invasion by the strains studied was not observed, suggesting that different bacterial and/or host cell factors are probably involved in these interactions.

Of the different well-characterized S. suis serotype 2 virulence factors, the CPS has, amongst its various properties, been described to modulate the adhesion to and invasion of epithelial and endothelial cells $[27,29,30$, 34]. Meanwhile, the SLY has more recently been suggested to be involved in the adhesion to and invasion of human epithelial cells at sub-cytolytic concentrations [35]. Finally, the roles of cell wall modifications, including the D-alanylation of the LTA and $\mathrm{N}$-acetylation of the PGN, in adhesion and invasion have been studied using pBMEC and porcine tracheal epithelial cells $[8$, 28]. Given these properties, the role of these factors on the adhesion to and invasion of meningeal cells by S. suis was determined. The adhesion of $S$. suis to meningeal cells was significantly modulated by the CPS $(\mathrm{p}<0.001)$ (Fig. 1C): the absence of the CPS increased adhesion by tenfold. Meanwhile, the SLY and cell wall modifications had no effect. The absence of the CPS also significantly increased invasion of meningeal cells (Fig. 1D), by one hundredfold ( $\mathrm{p}<0.001)$, such that the intracellular bacteria/cell ratio was of 10 . These modulations by the CPS, though expected since it is known to mask various surface proteins implicated in adhesion and invasion, are similar to those obtained using pBMEC and porcine tracheal epithelial cells $[11,28,34]$. In fact, P1/7 was non-invasive when encapsulated, but highly invasive in the absence of its CPS. Regulation of CPS expression may play an important role in bacterial-host interactions in vivo [1]. On the other hand, the SLY did not modulate the adhesion to and invasion of $\mathrm{pBMEC}$ or porcine tracheal epithelial cells by $S$. suis, as was observed in the present study [11, 28]. Meanwhile, the D-alanylation of the LTA modulated the adhesion to and invasion of pBMEC, but not of porcine tracheal epithelial cells $[8,11$, 28]. Consequently, different receptors may be implicated in adhesion and invasion according to the cell type and/ or species.

\section{Streptococcus suis serotype 2 adheres to and invades human astrocytes and these interactions are modulated by the CPS and, to a certain extent, by the SLY and cell wall modifications}

In the case where $S$. suis serotype 2 would modulate its CPS, the pathogen may penetrate the meningeal cells of the pia mater, as observed in this study, in order to reach the astrocytes of the glia limitans superficialis [21]. As with meningeal cells, no significant differences were observed between adhesion of the different STs to astrocytes. Indeed, adhesion ranged between $1 \times 10^{5}$ and $5 \times 10^{6} \mathrm{CFU}$, equivalent to 1 and 50 bacteria/cell, respectively (Fig. 2A). Moreover, the strains P1/7, LPH4 and 1088563 adhered similarly, but significantly less than the other strains $(\mathrm{p}<0.05)$. The adhesion levels of S. suis serotype 2 to human astrocytes are similar to those previously reported for GBS serotypes III and V [21].

Since all of the $S$. suis strains adhered to astrocytes, their subsequent invasion capacity was evaluated. Unlike with meningeal cells, multiple strains were, in theory, capable of biologically relevant invasion (Fig. 2B). Indeed, at least $1 \times 10^{5} \mathrm{CFU}$, equivalent to 1 intracellular bacterium/cell, were recovered following infection with the different strains, with the exception of P1/7 and 89-1591. Differences were nonetheless observed between the strains capable of invasion: 31533 and 1088563 invaded the cells significantly more than did SC84, LPH4 or MNCM43 ( $p<0.01$ ). These differences suggest that invasion of astrocytes by $S$. suis is a strain-specific characteristic: as mentioned previously however, the characteristics of ST25 and ST28 strains remain largely unknown. These results are different from those obtained using murine astrocytes, which did not internalize the $S$. suis serotype 2 strains 31533 (ST1) and SC84 (ST7), also used in this study [4]. On the contrary, invasion of human astrocytes by GBS was reported as infrequent since the intracellular bacterium/cell ratio recovered following infection was much lower than 1 [21]. As with meningeal cells however, S. suis adhesion to and invasion of astrocytes did not correlate. 


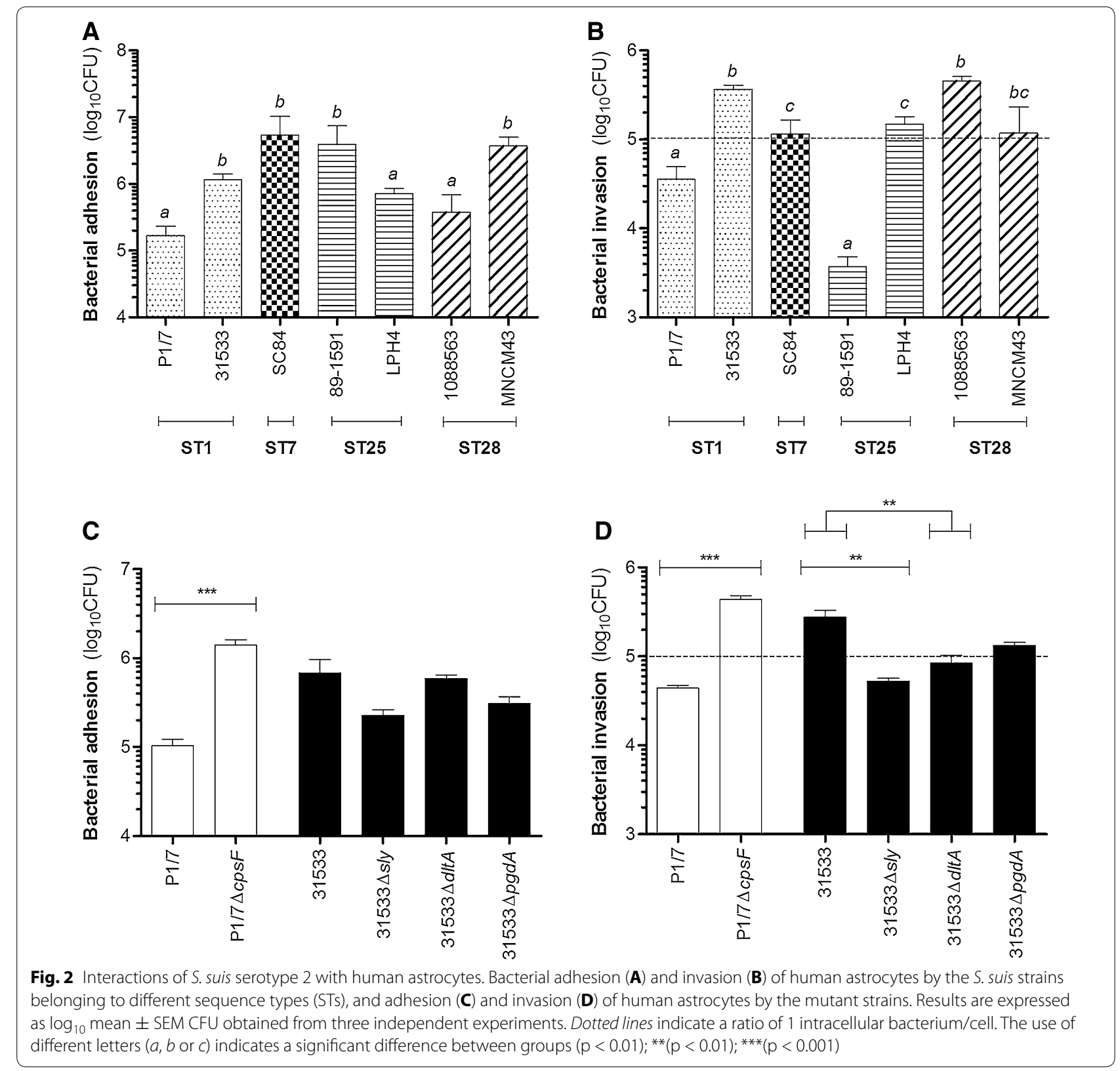

Since S. suis interacted with astrocytes, the role of the CPS, SLY and cell wall modifications were evaluated. Adhesion to human astrocytes was significantly modulated by the CPS ( $<$ < 0.001) (Fig. 2C), its absence increasing adhesion by tenfold. Meanwhile, the absence of the SLY and cell wall modifications did not modulate the adhesion of S. suis to astrocytes. The absence of the CPS also significantly increased the invasion capacity of astrocytes $(\mathrm{p}<0.001)$ (Fig. 2D), for which the intracellular bacteria/cell ratio recovered was of 5 , equivalent to a tenfold increase. This greatly differs from murine astrocytes, which S. suis did not invade even in the absence of CPS [4]. Unlike with meningeal cells however, the absence of the SLY and D-alanylation of the LTA significantly decreased invasion of astrocytes by $S$. suis $(\mathrm{p}<0.01)$ : the strain was no longer capable of biologically relevant cell invasion. Meanwhile, as with meningeal cells, the $\mathrm{N}$-acetylation of the PGN had no effect. These results are in accordance with previous studies: sub-cytolytic levels of SLY increased the invasion capacity of S. suis serotype 2 in epithelial cells [35], while, as mentioned above, the D-alanylation of the LTA modulated the invasion of pBMEC [8]. 


\section{Streptococcus suis serotype 2 induces little inflammatory response from infected human meningeal cells}

Streptococcus suis-induced inflammation is a characteristic of the infection that can lead to an exacerbated inflammatory response and host death [36]. Furthermore, meningitis, the hallmark of the $S$. suis CNS infection, is defined as an inflammation of the meninges in which the surrounding CNS tissue may be implicated [36]. Consequently, an understanding of the role of these cells in the production of inflammatory mediators following S. suis serotype 2 infection is crucial.

Human meningeal cells have been previously reported to produce the pro-inflammatory cytokine interleukin (IL) -6 and chemokines C-C motif ligand (CCL)2, CCL5 and $\mathrm{C}-\mathrm{X}-\mathrm{C}$ motif ligand $(\mathrm{CXCL}) 8$ in response to meningeal pathogens, but not IL-1 $\alpha$, IL-1 $\beta$, IL-10, IL-12, tumor necrosis factor (TNF)- $\alpha$, CCL3 or CCL4 [21-23]. Consequently, only the production of IL-6, CCL2, CCL5, and CXCL8 was quantified $24 \mathrm{~h}$ following infection with the different $S$. suis strains. Meningeal cells infected with $N$. meningitidis, used as a positive control, significantly produced all four mediators $(\mathrm{p}<0.01)$. However, no production of IL-6, CCL2 or CXCL8 was observed when cells were infected with $S$. suis, regardless of the strain (data not shown). Interestingly, a significant production of CCL5 was induced, but by both ST1 strains only $(\mathrm{p}<0.01)$ (Fig. 3). The levels were significantly greater

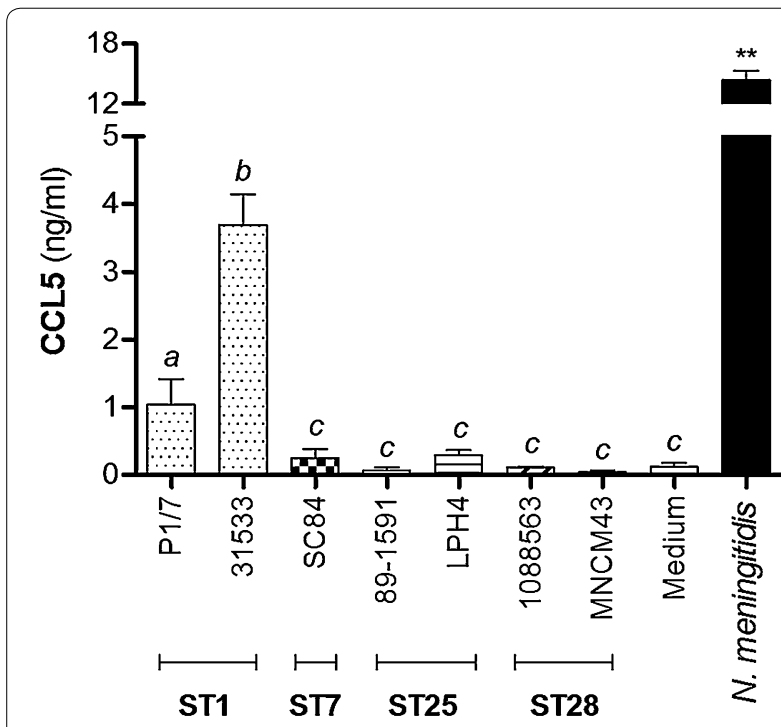

Fig. 3 Only S. suis serotype 2 ST1 strains induce CCL5 from infected human meningeal cells. CCL5 production from human meningeal cells infected with the $S$. suis strains belonging to different sequence types (STs). Results are expressed as mean $\pm \mathrm{SEM} \mathrm{ng} / \mathrm{ml}$ obtained from three independent experiments. The use of different letters $(a$, $b$ or $c)$ indicates a significant difference between groups ( $p<0.01)$. **Indicates a significant difference between $N$. meningitidis and the medium $(p<0.01)$ when cells were infected with 31533 than with P1/7 $(\mathrm{p}<0.01)$. This production of CCL5 corroborates with previous results in which elevated levels of the mediator were observed in the CSF of human patients with bacterial meningitis [37]. Furthermore, CCL5 is implicated in the chemoattraction of monocytes, which massively infiltrate the SAS and meninges during S. suis meningitis [36]. An absence of these four inflammatory mediators was also observed when human meningeal cells were infected with GBS or S. pneumoniae [21, 23]. By contrast, these cells produced important levels of the four mediators following infection with $N$. meningitidis and $H$. influenzae, but only produced CCL2 and CXCL8 following infection with E. coli [23]. However, S. suis serotype 2 induced IL-6, CCL2 and CXCL8 from hBMEC and IL-6 and CXCL8 from pBMEC, suggesting a possible cell and/ or species specificity [38, 39].

Given the relative absence of the inflammatory mediators evaluated, the S. suis-induced cytotoxicity was evaluated (Table 1). Cytotoxicity levels were below $10 \%$ following infection with the different strains, suggesting that unlike with the hBMEC and pBMEC, the SLY, produced by the ST1 and ST7 strains but not by the ST25 or ST28 strains, was not toxic for human meningeal cells. Consequently, the absence of the meningeal cell inflammatory mediators evaluated was probably not due to cell death. It is possible that human meningeal cells do not express certain of the various receptors implicated in the $S$. suis serotype 2 recognition, including TLRs (TLR2, TLR4, TLR9, and possibly, TLR3) and NLRs (NOD2) [40]. Of these however, the TLR2, often considered as the most important to the S. suis serotype 2 pathogenesis, and TLR4, are present on the cell surface $[1,41]$.

Table 1 Streptococcus suis serotype 2-induced human meningeal cell and astrocyte cytotoxicity, $24 \mathrm{~h}$ following infection

\begin{tabular}{|c|c|c|c|c|}
\hline \multirow[t]{2}{*}{ Strain } & \multirow[t]{2}{*}{$\begin{array}{l}\text { Sequence } \\
\text { type }\end{array}$} & \multirow[t]{2}{*}{$\begin{array}{l}\text { Presence } \\
\text { of SLY }\end{array}$} & \multicolumn{2}{|c|}{$\begin{array}{l}\% \text { cytotoxicity } \pm \text { SEM } \\
(\mathrm{n}=3)\end{array}$} \\
\hline & & & $\begin{array}{l}\text { Meningeal } \\
\text { cells }\end{array}$ & Astrocytes \\
\hline $\mathrm{P} 1 / 7$ & 1 & Yes & $0.1 \pm 0.1$ & $38.2 \pm 3.4$ \\
\hline 31533 & 1 & Yes & $3.4 \pm 1.2$ & $63.3 \pm 0.6$ \\
\hline SC84 & 7 & Yes & $9.1 \pm 2.6$ & $35.3 \pm 6.2$ \\
\hline 89-1591 & 25 & No & $0.0 \pm 0.0$ & $71.5 \pm 1.5$ \\
\hline LPH4 & 25 & No & $6.1 \pm 2.2$ & $28.9 \pm 6.0$ \\
\hline 1088563 & 28 & No & $0.0 \pm 0.0$ & $12.0 \pm 3.9$ \\
\hline MNCM43 & 28 & No & $0.0 \pm 0.0$ & $1.9 \pm 1.0$ \\
\hline $\begin{array}{l}\text { N. menin- } \\
\text { gitidis }\end{array}$ & $\begin{array}{l}\text { Not applica- } \\
\text { ble }\end{array}$ & $\begin{array}{l}\text { Not applica- } \\
\text { ble }\end{array}$ & $\begin{array}{l}\text { Not deter- } \\
\text { mined }\end{array}$ & $88.6 \pm 0.9$ \\
\hline
\end{tabular}




\section{Streptococcus suis serotype 2 does not induce an inflammatory response from infected human astrocytes}

Human astrocytes produce various inflammatory mediators following bacterial infection, including IL-6, CCL2, CCL5, and CXCL8 [21]. Consequently, these mediators were quantified following infection with the different $S$. suis strains. As with meningeal cells, $N$. meningitidis induced a significant production of all four mediators $(\mathrm{p}<0.01)$. Meanwhile, no production of IL-6, CCL2, CCL5 or CXCL8 was observed when cells were infected with S. suis, regardless of the strain (data not shown). Interestingly, these results vary from those obtained using murine astrocytes, which were an important source of TNF- $\alpha$, IL-6, CCL2, and CXCL1 following $S$. suis serotype 2 infection [4]. Furthermore, recognition of $S$. suis serotype 2 by murine astrocytes was mainly TLR2-dependent, since the expression of TLR1, TLR4 and TLR6 was barely modulated following infection [4]. Meanwhile $S$. suis serotype 2 -induced TNF- $\alpha$ and CCL2 were partially dependent on TLR2 in murine astrocytes [4]. By contrast, in the present study, no CCL2 production was observed when human astrocytes were infected with S. suis serotype 2, while production of TNF- $\alpha$ was not determined. Consequently, the species from which the cells originate appears to play an important role on the results obtained, as the receptors present may vary, which could influence the subsequent inflammatory response [42, 43].

Given the absence of the inflammatory mediators measured, S. suis-induced astrocyte cytotoxicity was also evaluated (Table 1 ). In contrast to the lack of meningeal cell death following infection with S. suis, astrocytes were generally more sensitive to infection. The ST1, ST7 and ST25 strains induced important cytotoxicity, which ranged between approximately 30 and $70 \%$, while the ST28 strains caused little cytotoxicity (less than $15 \%)$. The SLY may be partially responsible for the S. suis-induced astrocyte cell death since the ST1 and ST7 strains induced higher cytotoxicity levels than did the SLY-negative strain LPH4. Furthermore, the differences observed between the ST1 and ST25 strains suggest that strain-specific factors are implicated in these interactions. Nevertheless, the $S$. suis-induced astrocyte cell death was probably not responsible for the absence of the inflammatory mediators evaluated, since N. meningitis caused near total cell death, with $90 \%$ cytotoxicity, yet induced important levels of these inflammatory mediators.

\section{Conclusions}

Streptococcus suis serotype 2 interacted with human meningeal cells and astrocytes via the adhesion to both cell types; invasion of meningeal cells was rare however, whereas that of astrocytes was generally more frequent. Furthermore, these interactions were largely modulated by the CPS, and to a certain extent, by the SLY and cell wall modifications. Regardless of the fact that S. suis interacted with meningeal cells and astrocytes, little or no production of the inflammatory mediators evaluated was observed. This suggests that the meningeal cells of the leptomeninges and the astrocytes of the underlying glia limitans superficialis may not be directly implicated in the inflammatory response observed during S. suis meningitis in humans. Nevertheless, the use of a microarray or proteomic assay investigating a larger number and greater variety of inflammatory mediators induced by $S$. suis serotype 2 in these cell types may yield further details. Alongside, a given ST could not be correlated with a specific interaction since important variations were observed between strains within a single ST. Consequently, the interactions with meningeal cells and astrocytes, though important for the pathogenesis, are not ST-dependent but rather a characteristic of S. suis serotype 2 that varies according to the strain.

\section{Methods}

\section{Bacterial strains and growth conditions}

The well-encapsulated $S$. suis serotype 2 strains and isogenic mutants for different well-characterized virulence factors used in this study are listed in Table 2. Bacteria were grown overnight on Columbia Blood Agar containing $5 \%$ sheep blood (v/v) at $37{ }^{\circ} \mathrm{C}$ with $5 \% \mathrm{CO}_{2}$ (Oxoid, Basingstoke, UK). Five $\mathrm{ml}$ of Todd-Hewitt Broth (THB; Becton-Dickinson, Swindon, UK) were inoculated and incubated for $8 \mathrm{~h}$ at $37{ }^{\circ} \mathrm{C}$ with $5 \% \mathrm{CO}_{2}$. Working cultures were prepared by inoculating $30 \mathrm{ml}$ of THB with $10 \mu \mathrm{l}$ of a $10^{-3}$ dilution of the $8 \mathrm{~h}$ cultures, incubated for $16 \mathrm{~h}$ at $37{ }^{\circ} \mathrm{C}$ with $5 \% \mathrm{CO}_{2}$. Bacteria were washed twice with $\mathrm{pH}$ 7.3 phosphate-buffered saline (PBS), resuspended in cell culture medium, appropriately diluted, and plated on Todd-Hewitt Broth agar (THA) to accurately determine concentrations. The $N$. meningitidis serogroup B strain MC58 [44], used as a positive control, was grown on supplemented GC agar and bacterial suspensions prepared in cell culture medium, as previously described [24].

\section{Human meningeal cell and astrocyte cultures}

Human meningioma cells, obtained from surgically removed tumors as previously described, express the characteristic markers of desmosomal desmoplakin, epithelial membrane antigen, vimentin, and cytokeratin [24]. The cells were grown in Dulbecco's Modified Eagle Medium containing Glutamax-1 and sodium pyruvate (Lonza, Slough, UK) supplemented with $10 \%$ fetal calf serum (v/v) (FCS; Lonza) and seeded in flasks precoated with $5 \mathrm{mg} / \mathrm{cm}^{2}$ of type I collagen from rat tail 
Table 2 Streptococcus suis serotype 2 strains used in this study

\begin{tabular}{|c|c|c|c|c|c|}
\hline Strain & Sequence type & Country & Host & Phenotype & References \\
\hline P1/7 & 1 & United Kingdom & Pig & Wild-type & {$[46]$} \\
\hline $\mathrm{P} 1 / 7 \triangle c p s F$ & 1 & - & - & Non-encapsulated mutant & {$[6]$} \\
\hline 31533 & 1 & France & Pig & Wild-type & {$[47]$} \\
\hline $31533 \triangle s / y$ & 1 & - & - & Suilysin-deficient mutant & {$[7]$} \\
\hline $31533 \triangle d l t A$ & 1 & - & - & D-alanylation of lipoteichoic acid-deficient mutant & {$[8]$} \\
\hline $31533 \triangle p g d A$ & 1 & - & - & N-deacetylation of peptidoglycan-deficient mutant & {$[9]$} \\
\hline SC84 & 7 & China & Human & Wild-type & {$[48]$} \\
\hline $89-1591$ & 25 & Canada & Pig & Wild-type & {$[49]$} \\
\hline $\mathrm{LPH} 4$ & 25 & Thailand & Human & Wild-type & {$[50]$} \\
\hline 1088563 & 28 & Canada & Pig & Wild-type & [51] \\
\hline MNCM43 & 28 & Thailand & Human & Wild-type & {$[50]$} \\
\hline
\end{tabular}

(Becton-Dickinson); culture passages 2-10 were used [24]. SVGmm human fetal astrocyte cells were grown in Eagle's Minimal Essential Medium (Lonza) supplemented with $10 \%$ FCS (v/v) [45].

\section{Infection of human meningeal cells and astrocytes}

Human meningeal cells and astrocytes were grown to confluence in 24-well cell culture plates, averaging $4 \times 10^{4}$ cells/well and $1 \times 10^{5}$ cells/well, respectively. Cell monolayers were maintained overnight in medium containing $1 \%$ FCS (v/v) and washed twice with warm PBS prior to bacterial challenge, in triplicate, with the different $S$. suis serotype 2 wild-type or mutant strains, or $N$. meningitidis. Based on preliminary assays, $1 \mathrm{ml}$ of $4 \times 10^{4} \mathrm{CFU} /$ well for meningeal cells and $1 \times 10^{5} \mathrm{CFU} /$ well for astrocytes [multiplicity of infection $(\mathrm{MOI})=1$ ] was added for bacterial adhesion. For bacterial invasion, host cell cytotoxicity and inflammatory activation, $4 \times 10^{6} \mathrm{CFU} /$ well for meningeal cells and $1 \times 10^{7} \mathrm{CFU} /$ well for astrocytes $(\mathrm{MOI}=100)$ were added. Monolayers were incubated for $9 \mathrm{~h}$ (adhesion and invasion) or $24 \mathrm{~h}$ (cell cytotoxicity and inflammatory activation) at $37{ }^{\circ} \mathrm{C}$ with $5 \% \mathrm{CO}_{2}$ as previously described [21-23].

\section{Bacterial adhesion and invasion measurement}

After incubation, cells were washed four times with warm PBS, lysed using $250 \mu \mathrm{l} /$ well of $1 \%$ saponin (w/v) (Sigma-Aldrich, Dorset, UK) in PBS and incubated for 15 min at $37{ }^{\circ} \mathrm{C}$ with $5 \% \mathrm{CO}_{2}$. Bacterial adhesion was measured by plating the lysates onto THA and incubating overnight at $37{ }^{\circ} \mathrm{C}$ with $5 \% \mathrm{CO}_{2}$. Bacterial invasion was carried out using the antibiotic protection assay [5]: cell medium was removed after $9 \mathrm{~h}$, monolayers were washed twice with warm PBS and $1 \mathrm{ml}$ of medium containing $5 \mu \mathrm{g} / \mathrm{ml}$ penicillin G (Sigma-Aldrich) and $100 \mu \mathrm{g} /$ $\mathrm{ml}$ of gentamicin (Sigma-Aldrich) was added for $90 \mathrm{~min}$ at $37{ }^{\circ} \mathrm{C}$ with $5 \% \mathrm{CO}_{2}$ to kill extracellular bacteria. The last wash was plated to confirm antibiotic activity.

\section{Host cell cytotoxicity assay}

Lactate dehydrogenase (LDH) release was measured $24 \mathrm{~h}$ post-infection using the CytoTox 96 Non-Radioactive Cytotoxicity Assay kit (Promega, UK) according to instructions. Non-infected monolayers were included for measurement of spontaneous LDH release and maximum LDH release was induced using the lysis reagent included in the kit. The absorbance was read at $492 \mathrm{~nm}$ using the iMark Absorbance Reader (Bio-Rad, Hercules, CA, USA).

\section{Host cell inflammatory activation}

The measurement of the pro-inflammatory cytokine IL-6 and chemokines CCL2, CCL5 and CXCL8 was carried out by enzyme-linked immunosorbent assay as previously described [22].

\section{Statistical analyses}

Unpaired t-tests, Mann-Whitney rank sum tests and one-way ANOVA, where appropriate, were performed to find statistical differences between groups. $\mathrm{p}<0.05$ was considered statistically significant.

\section{Abbreviations}

CCL: C-C motif ligand; CFU: colony forming unit; CNS: central nervous system; CPEC: choroid plexus epithelial cells; CPS: capsular polysaccharide; CSF: cerebrospinal fluid; CXCL: C-X-C motif ligand; FCS: fetal calf serum; GBS: group B Streptococcus; hBMEC: human brain microvascular endothelial cells; IL: interleukin; LDH: lactate dehydrogenase; LTA: lipoteichoic acid; MOI: multiplicity of infection; NOD: nucleotide-binding oligomerization domain; NLR: NOD-like receptors; PBMEC: porcine brain microvascular endothelial cells; PBS: phosphate-buffered saline; PGN: peptidoglycan; SAS: subarachnoid space; SLY: suilysin; ST: sequence type; THA: Todd-Hewitt Broth agar; THB:Todd-Hewitt Broth; TLR: toll-like receptor; TNF: tumor necrosis factor; v/v: volume/volume; w/v: weight/volume. 


\section{Authors' contributions}

JPA carried out the experiments described in this study. MG, MC, MS, and JX participated in the supervision and conception of this study. JPA prepared the manuscript under the supervision of MG. All authors read and approved the manuscript.

\section{Author details \\ 1 Faculty of Veterinary Medicine, Research Group on Infectious Diseases of Swine (GREMIP) and Swine and Poultry Infectious Diseases Research Center (CRIPA), University of Montreal, 3200 Sicotte Street, Saint-Hyacinthe, QC J2S 2M2, Canada. ${ }^{2}$ Neisseria Research Group, Molecular Microbiology, Clinical and Experimental Sciences, University of Southampton Faculty of Medicine, Southampton, UK. ${ }^{3}$ Collaborative Innovation Center for Diagnosis and Treat- ment of Infectious Diseases, National Institute for Communicable Disease Control and Prevention, Chinese Center for Disease Control and Prevention, Beijing, China.}

\section{Acknowledgements}

This study was funded in part by the Canadian Institutes of Health Research (CIHR) China-Canada Joint Health Research Initiative to MG, MS and JX, and by Meningitis UK to MC. International internship scholarships to study in the United Kingdom (MC) were made possible by the Fond de recherche du Québec-Nature et technologie (FRQ-NT) and the University of Montreal to JPA. JPA is the recipient of a FRQ-NT doctoral award.

\section{Competing interests}

The authors declare that they have no competing interests.

Received: 21 May 2015 Accepted: 14 October 2015

Published online: 26 October 2015

\section{References}

1. Fittipaldi N, Segura M, Grenier D, Gottschalk M. Virulence factors involved in the pathogenesis of the infection caused by the swine pathogen and zoonotic agent Streptococcus suis. Future Microbiol. 2012;7(2):259-79.

2. Goyette-Desjardins G, Auger JP, Xu J, Segura M, Gottschalk M. Streptococcus suis, an important pig pathogen and emerging zoonotic agentan update on the worldwide distribution based on serotyping and sequence typing. Emerg Microbes Infect. 2014;3(6):e45.

3. Zheng H, Luo X, Segura M, Sun H, Ye C, Gottschalk M, et al. The role of toll-like receptors in the pathogenesis of Streptococcus suis. Vet Microbiol. 2012;156(1-2):147-56.

4. Zheng H, Punaro MC, Segura M, Lachance C, Rivest S, Xu J, et al. Toll-like receptor 2 is partially involved in the activation of murine astrocytes by Streptococcus suis, an important zoonotic agent of meningitis. J Neuroimmunol. 2011;234(1-2):71-83.

5. Segura MA, Cleroux P, Gottschalk M. Streptococcus suis and group B Streptococcus differ in their interactions with murine macrophages. FEMS Immunol Med Microbiol. 1998;21(3):189-95.

6. Lecours MP, Gottschalk M, Houde M, Lemire P, Fittipaldi N, Segura M. Critical role for Streptococcus suis cell wall modifications and suilysin in resistance to complement-dependent killing by dendritic cells. J Infect Dis. 2011;204(6):919-29.

7. Lun S, Perez-Casal J, Connor W, Willson PJ. Role of suilysin in pathogenesis of Streptococcus suis capsular serotype 2. Microb Pathog. 2003;34(1):27-37.

8. Fittipaldi N, Sekizaki T, Takamatsu D, Harel J, Dominguez-Punaro Mde L, Von Aulock S, et al. D-alanylation of lipoteichoic acid contributes to the virulence of Streptococcus suis. Infect Immun. 2008;76(8):3587-94.

9. Fittipaldi N, Sekizaki T, Takamatsu D, de la Cruz Dominguez-Punaro M, Harel J, Bui NK, et al. Significant contribution of the pgdA gene to the virulence of Streptococcus suis. Mol Microbiol. 2008;70(5):1120-35.

10. de la Dominguez-Punaro $M$, Segura M, Contreras I, Lachance C, Houde M, Lecours MP, et al. In vitro characterization of the microglial inflammatory response to Streptococcus suis, an important emerging zoonotic agent of meningitis. Infect Immun. 2010;78(12):5074-85.
11. Vanier G, Segura M, Friedl P, Lacouture S, Gottschalk M. Invasion of porcine brain microvascular endothelial cells by Streptococcus suis serotype 2. Infect Immun. 2004;72(3):1441-9.

12. Tenenbaum T, Adam R, Eggelnpohler I, Matalon D, Seibt A, Novotny $\mathrm{GE}$, et al. Strain-dependent disruption of blood-cerebrospinal fluid barrier by Streptoccocus suis in vitro. FEMS Immunol Med Microbiol. 2005; 44(1):25-34

13. Charland N, Nizet V, Rubens CE, Kim KS, Lacouture S, Gottschalk M. Streptococcus suis serotype 2 interactions with human brain microvascular endothelial cells. Infect Immun. 2000;68(2):637-43.

14. Schwerk C, Papandreou T, Schuhmann D, Nickol L, Borkowski J, Steinmann $U$, et al. Polar invasion and translocation of Neisseria meningitidis and Streptococcus suis in a novel human model of the blood-cerebrospinal fluid barrier. PLoS One. 2012;7(1):e30069.

15. Alcolado R, Weller RO, Parrish EP, Garrod D. The cranial arachnoid and pia mater in man: anatomical and ultrastructural observations. Neuropathol Appl Neurobiol. 1988;14(1):1-17.

16. Engelhardt B, Coisne C. Fluids and barriers of the CNS establish immune privilege by confining immune surveillance to a two-walled castle moat surrounding the CNS castle. Fluids Barriers CNS. 2011;8(1):4.

17. Sofroniew MV, Vinters HV. Astrocytes: biology and pathology. Acta Neuropathol. 2010;119(1):7-35.

18. Farina C, Aloisi F, Meinl E. Astrocytes are active players in cerebral innate immunity. Trends Immunol. 2007;28(3):138-45.

19. Dando SJ, Mackay-Sim A, Norton R, Currie BJ, St John JA, Ekberg JA, et al. Pathogens penetrating the central nervous system: infection pathways and the cellular and molecular mechanisms of invasion. Clin Microbiol Rev. 2014;27(4):691-726.

20. Streptococcosis Gottschalk M. In: Zimmerman JJ, Ramirez A, Schwartz KJ, Stevenson GW, editors. Diseases of swine. 10th ed. Ames: Wiley-Blackwell Publishing; 2012. p. 841-55.

21. Alkuwaity K, Taylor A, Heckels JE, Doran KS, Christodoulides M. Group B Streptococcus interactions with human meningeal cells and astrocytes in vitro. PLoS One. 2012;7(8):e42660.

22. Christodoulides M, Makepeace BL, Partridge KA, Kaur D, Fowler MI, Weller $\mathrm{RO}$, et al. Interaction of Neisseria meningitidis with human meningeal cells induces the secretion of a distinct group of chemotactic, proinflammatory, and growth-factor cytokines. Infect Immun. 2002;70(8):4035-44.

23. Fowler MI, Weller RO, Heckels JE, Christodoulides M. Different meningitiscausing bacteria induce distinct inflammatory responses on interaction with cells of the human meninges. Cell Microbiol. 2004;6(6):555-67.

24. Hardy SJ, Christodoulides M, Weller RO, Heckels JE. Interactions of Neisseria meningitidis with cells of the human meninges. Mol Microbiol. 2000;36(4):817-29.

25. Kim KS. Pathogenesis of bacterial meningitis: from bacteraemia to neuronal injury. Nat Rev Neurosci. 2003;4(5):376-85.

26. Feurer DJ, Weller RO. Barrier functions of the leptomeninges: a study of normal meninges and meningiomas in tissue culture. Neuropathol Appl Neurobiol. 1991;17(5):391-405.

27. Lalonde M, Segura M, Lacouture S, Gottschalk M. Interactions between Streptococcus suis serotype 2 and different epithelial cell lines. Microbiology. 2000;146(8):1913-21.

28. Wang Y, Gagnon CA, Savard C, Music N, Srednik M, Segura M, et al. Capsular sialic acid of Streptococcus suis serotype 2 binds to swine influenza virus and enhances bacterial interactions with virus-infected tracheal epithelial cells. Infect Immun. 2013;81(12):4498-508.

29. Ferrando ML, de Greeff A, van Rooijen WJ, Stockhofe-Zurwieden N, Nielsen J, Wichgers Schreur PJ, et al. Host-pathogen interaction at the intestinal mucosa correlates with zoonotic potential of Streptococcus suis. J Infect Dis. 2015;212(1):95-105.

30. Benga L, Friedl P, Valentin-Weigand P. Adherence of Streptococcus suis to porcine endothelial cells. J Vet Med B Infect Dis Vet Public Health. 2005;52(9):392-5.

31. Moxon ER, Murphy PA. Haemophilus influenzae bacteremia and meningitis resulting from survival of a single organism. Proc Natl Acad Sci USA. 1978;75(3):1534-6.

32. Ye C, Zheng $H$, Zhang J, Jing $H$, Wang L, Xiong $Y$, et al. Clinical, experimental, and genomic differences between intermediately pathogenic, highly pathogenic, and epidemic Streptococcus suis. J Infect Dis. 2009;199(1):97-107. 
33. Tenenbaum T, Papandreou T, Gellrich D, Friedrichs U, Seibt A, Adam $R$, et al. Polar bacterial invasion and translocation of Streptococcus suis across the blood-cerebrospinal fluid barrier in vitro. Cell Microbiol. 2009;11(2):323-36.

34. Benga L, Goethe R, Rohde M, Valentin-Weigand P. Non-encapsulated strains reveal novel insights in invasion and survival of Streptococcus suis in epithelial cells. Cell Microbiol. 2004;6(9):867-81.

35. Seitz M, Baums CG, Neis C, Benga L, Fulde M, Rohde M, et al. Subcytolytic effects of suilysin on interaction of Streptococcus suis with epithelial cells. Vet Microbiol. 2013;167(3-4):584-91.

36. Dominguez-Punaro MC, Segura M, Plante MM, Lacouture S, Rivest S, Gottschalk M. Streptococcus suis serotype 2, an important swine and human pathogen, induces strong systemic and cerebral inflammatory responses in a mouse model of infection. J Immunol. 2007;179(3):1842-54.

37. Lahrtz F, Piali L, Spanaus KS, Seebach J, Fontana A. Chemokines and chemotaxis of leukocytes in infectious meningitis. J Neuroimmunol. 1998;85(1):33-43.

38. Vanier G, Segura M, Lecours MP, Grenier D, Gottschalk M. Porcine brain microvascular endothelial cell-derived interleukin-8 is first induced and then degraded by Streptococcus suis. Microb Pathog. 2009;46(3):135-43.

39. Vadeboncoeur N, Segura M, Al-Numani D, Vanier G, Gottschalk M. Proinfammatory cytokine and chemokine release by human brain microvascular endothelial cells stimulated by Streptococcus suis serotype 2. FEMS Immunol Med Microbiol. 2003;35(1):49-58.

40. Lecours MP, Segura M, Fittipaldi N, Rivest S, Gottschalk M. Immune receptors involved in Streptococcus suis recognition by dendritic cells. PLoS One. 2012;7(9):e44746.

41. Humphries HE, Triantafilou M, Makepeace BL, Heckels JE, Triantafilou K, Christodoulides M. Activation of human meningeal cells is modulated by lipopolysaccharide (LPS) and non-LPS components of Neisseria meningitidis and is independent of Toll-like receptor (TLR)4 and TLR2 signalling. Cell Microbiol. 2005;7(3):415-30.

42. Tarassishin L, Suh HS, Lee SC. LPS and IL-1 differentially activate mouse and human astrocytes: role of CD14. Glia. 2014;62(6):999-1013.

43. Mestas J, Hughes CCW. Of mice and not men: differences between mouse and human immunology. J Immunol. 2004;172(5):2731-8.

44. McGuinness BT, Clarke IN, Lambden PR, Barlow AK, Poolman JT, Jones $\mathrm{DM}$, et al. Point mutation in meningococcal porA gene associated with increased endemic disease. Lancet. 1991;337(8740):514-7.

45. Major EO, Miller AE, Mourrain P, Traub RG, de Widt E, Sever J. Establishment of a line of human fetal glial cells that supports JC virus multiplication. Proc Natl Acad Sci USA. 1985;82(4):1257-61.

46. Slater JD, Allen AG, May JP, Bolitho S, Lindsay H, Maskell DJ. Mutagenesis of Streptococcus equi and Streptococcus suis by transposon Tn917. Vet Microbiol. 2003;93(3):197-206.

47. Kobisch M, Gottschalk M, Morvan M, Cariolet G, Bénévent G, Joly JP. Experimental infection of SPF piglets with Streptococcus suis serotype 2. J Rech Porcine Fr. 1995;27:97-102.

48. Ye C, Zhu X, Jing H, Du H, Segura M, Zheng H, et al. Streptococcus suis sequence type 7 outbreak, Sichuan, China. Emerg Infect Dis. 2006;12(8):1203-8.

49. Gottschalk M, Higgins R, Jacques M, Dubreuil D. Production and characterization of two Streptococcus suis capsular type 2 mutants. Vet Microbiol. 1992;30(1):59-71.

50. Takamatsu D, Wongsawan K, Osaki M, Nishino H, Ishiji T, Tharavichitkul P, et al. Streptococcus suis in humans, Thailand. Emerg Infect Dis. 2008;14(1):181-3.

51. Fittipaldi N, Xu J, Lacouture S, Tharavichitkul P, Osaki M, Sekizaki T, et al. Lineage and virulence of Streptococcus suis serotype 2 isolates from North America. Emerg Infect Dis. 2011;17(12):2239-44.

\section{Submit your next manuscript to BioMed Central and take full advantage of:}

- Convenient online submission

- Thorough peer review

- No space constraints or color figure charges

- Immediate publication on acceptance

- Inclusion in PubMed, CAS, Scopus and Google Scholar

- Research which is freely available for redistribution

Submit your manuscript at

www.biomedcentral.com/submit

C BioMed Central 